\title{
Airborne LiDAR
}

\author{
Jagdeep Kaur ${ }^{1}$, Harsh Jindal ${ }^{*}$ \\ ${ }^{1}$ Department of Physics, Chandigarh University, Punjab, India \\ ${ }^{1 *}$ Department of Computer Science Engineering, Chandigarh University, Punjab, India
}

\begin{abstract}
Article Info

Volume 8, Issue 3

Page Number: 495-498

Publication Issue :

May-June-2021

Article History

Accepted : 18 June 2021

Published: 25 June 2021

This data has been used in many existing inventions and even in many new inventions. This paper consists of a review of the current state of LiDAR technology and covers issues related to both data capturing and processing. In this paper, a discussion on different types of LiDAR sensors including LiDAR for autonomous vehicles was also done. It also explains existing data techniques and also gives a view of CNN (Convolutional Neural Networks). This paper also discusses the autonomous LiDAR technique in detail. The paper also discusses the future scope of technology.

Keywords : Lidar, CNN, Air-borne technology

Abbreviation-

PRF- Pulse Repetition Frequency GPS- Global Positioning System LML- Linear Mode LIDAR

GNSS- Global Navigation Satellite System SPL-Single Photon LIDAR
\end{abstract}

\section{INTRODUCTION}

The primary goal of the present study is having a detailed review of the LiDAR technology and to focus on various problems which are related to this particular technology as well as identifying the position where the technology is leading to. There will be different aspect in which this review will be carried out and that is gathering data, processing of data, analysing the accuracy, classification of data, data visualization and lastly realising the applications and the technology's prospects. There are various areas where the work can be done for LiDAR inventions and these areas are drones, space vehicles, ground-based vehicles, aircraft and helicopters, and tripods.

Data capture methods, data analysis procedures, implementations of data vary, however, the fundamentals are similar in any situation. Another important point about this topic is that it relies on hovering LiDAR automation. Following parts of this topic will provide some particulars on the concepts of technology, different kinds of magnetic eyes, elements for flight field, file and data formats, precise data measurement, classification systems, point cloud visualisation, implementation fields and future reach. Infra-red light is used by LiDAR, visible light by 
cameras and is thus more sensitive to rain, clouds, or textures. Furthermore, they focus on the fact that LiDAR is a shortcut and is only capable of processing spatial information, not the highway environment's complexities. LiDAR is rendered to be obsolete, as when the cameras are combined to simple conventional radar, many factors are listed for addressing the limitations of the system when used in adverse conditions.

\section{Principle of Lidar Technology}

A system of airborne LiDAR is comprised of the following components:

(a) A platform for air-borne technology that are used for flying a LiDAR sensor over the interested area;

(b) The LiDAR sensor used for generating laser over a shorter width transmits it to the ground, scans the surface by firing the pulses, and further receives the return signal.

(c) The GNSS receives, operating with the help of a tandem at the GNSS base station receiver based at the ground, functions by observing the aircraft position on every GNSS Epoch.

(d) IMU sensors used to observe the orientation and acceleration of an aircraft on a frequency higher than the GNSS Epoch.

(e) A computer onboard, responsible for timestamping the different data which is produced by the sensors attached above with the help of GNSS time, and the raw data is then achieved. This is also another common method where a digital camera is flown in a medium attached with LiDAR sensors as the colour information of terrain is provided by these cameras.

\section{TYPES OF LIDAR}

LML

In this type of sensors, only one pule is received \& is transferred to a sensor. Two consecutive pulses were separated with an adequate time gap to transmit the next pulse only after the first or the previous pulse is received. It is possible to transmit PRF (Pulse Repetition Frequency) of $400 \mathrm{kHz}$ order using the present-day scanner sold in the market. However, these scanners hold a limitation in terms of the altitude of flying were more the altitude, the lesser is the range of Pulse Repetition Frequency and the energies of the waves received at the receiver diminishes. Such sensors work well for less area.

\section{MPiA}

In the above discussed single pulse LML, there is a sequential transmission of pulses, but was not able to differentiate between the pulses. However, this limits the flying altitude and Pulse Repetition Frequency. Using the FWD data is dependent on the application. Many sensors also support FWD.

\section{MSL}

The intensity of the pulse of return signals helps in classifying the LiDAR data, which is the same as that of the case of the images. Because of this, the same was also investigated widely by many researchers specifically for the work on return intensity normalization on different angles and range.

\section{SPL and GML}

A laser pulse is split into multiple pulses. Generally, these sensors are cost-efficient for the surface area beyond 200square $\mathrm{km}$.

\section{RESULT}

LiDAR technology is not the only self-driven detection system, attached with cameras as the famous company Tesla, is the main provider in the field providing several application and advantages which are not possible to list in brief. LiDAR technology is far better, with the creation of 3-D cloud points, used in judging distances covered instead of using conventional cameras, along with this they are impervious to surfaces that are texture less, reflective, and textured. The data from LiDAR can 
represent and capture the physical environment around us like never before. The technology can be applied to other systems like sound propagation modelling, flood modelling, electromagnetic wave propagation modelling, and many others. However, still, numerous issues prevail with the usage of this technology where a LiDAR could be applied to find, availability analysis of sunlight, propagation of airpollution, analysis of air movement in a corridor, in the urban environment setups. The programme of 3D elevation of USGS for the U.S. is another important region where the data from LiDAR technology could be expected to play a major part. Generating a large LiDAR volume of data, and in some areas, the time lapse of facts is also required in checking the development of techniques involving better processing.

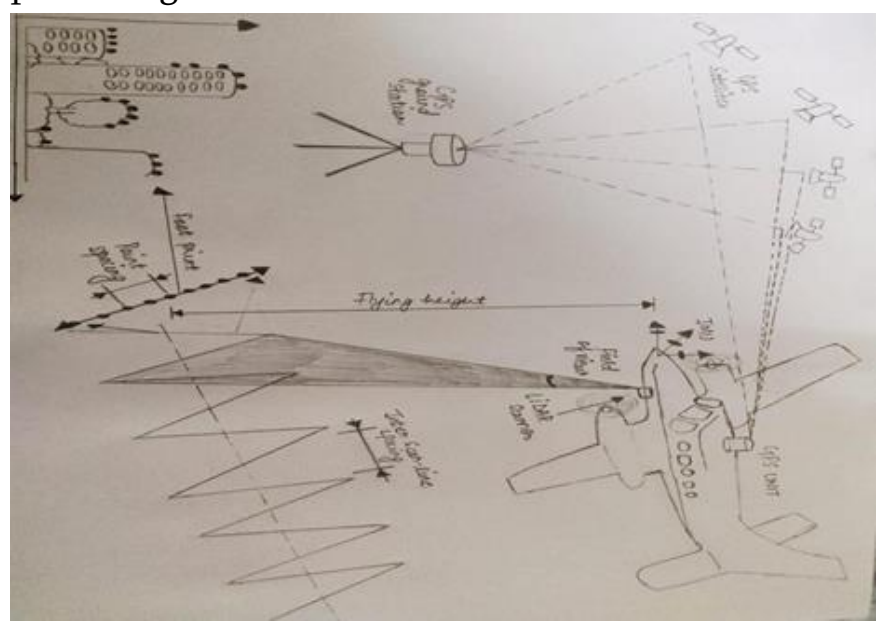

Figure 1: The use of LIDAR

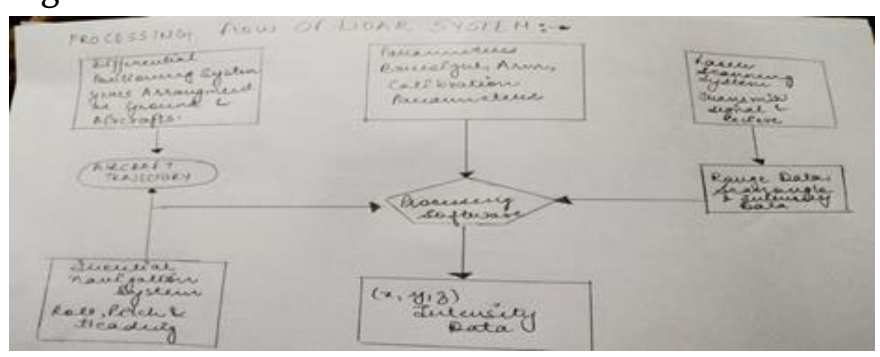

Figure 2: The processing steps for different data collected from the sensor is shown

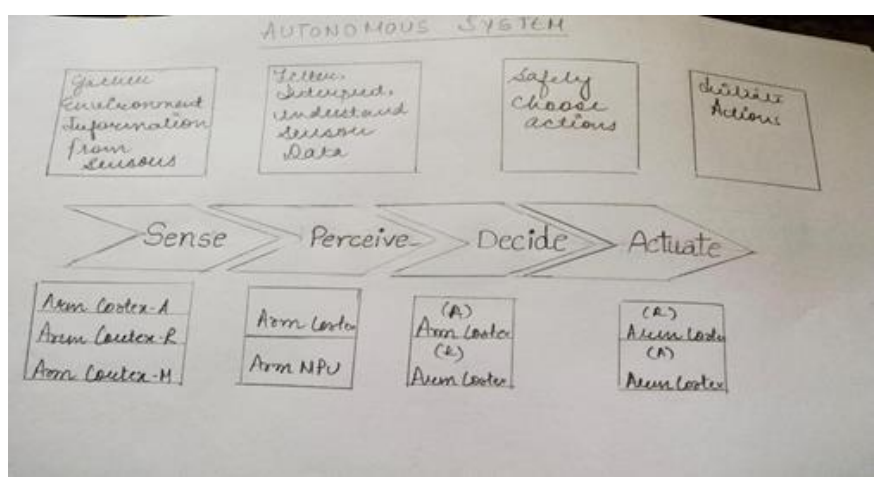

Figure 3: The exact aircraft position was estimated by the differentiation process of reference and on-board receivers. For the process of autonomous vehicles

\section{DISCUSSION}

The basic and foremost criterion of the paper is to discuss the defects in the LiDAR technology used in recent times in flights and other areas. The principles of LiDAR are yet to be used more efficiently in the inventions present and to be found. The paper also shows there is even much more to explore in LiDAR in upcoming times.

\section{CONCLUSION}

The paper presents review on LiDAR, its benefits and its issues and the method to creative methodology to create less expenditure for project. The paper also has mentioned the accuracy and quality of LiDAR. The paper also discusses its uses and future scope. In fact, LiDAR can be the next futuristic advancement of today's sensing field.

\section{REFERENCES}

[1]. Brovelli MA, Cannata M, \& Longoni UM (2004) LIDAR Data Filtering and DTM Interpolation Within GRASS.

[2]. Lidar, D. A. (2019). Arbitrary-time error suppression for Markovian adiabatic quantum computing using stabilizer subspace codes. PHYSICAL REVIEW A, 100(2). https://doi.org/10.1103/PhysRevA.100.022326 
[3]. Lemos, P. A., Franken, M., Mariani Jr., J., Pitta, F. G., Oliveira, F. A. P., Cunha-Lima, G., Caixeta, A. M., Almeida, B. O., \& Garcia, R. G. (n.d.). Use of robotic assistance to reduce proximity and air-sharing during percutaneous cardiovascular intervention. FUTURE CARDIOLOGY. https://doi.org/10.2217/fca2021-0024

[4]. James, S., \& Raheb, R. (2019). Quantum Computing Landing Aircraft. 2019 INTEGRATED COMMUNICATIONS, NAVIGATION AND SURVEILLANCE CONFERENCE (ICNS).

[5]. Khan, H. N., Chaudhuri, A., Das, A., \& Chaudhuri, A. (2020). An ultra robust session key based image cryptography. MICROSYSTEM TECHNOLOGIES-MICROAND NANOSYSTEMS-INFORMATION STORAGE AND PROCESSING SYSTEMS, 26(7, SI), 2193-2201.

https://doi.org/10.1007/s00542-019-04518-9

[6]. Advances in Airborne Lidar Systems and Data Processing

[7]. LiDAR Technologies and Systems

[8]. Dashora A, Lohani B, \& Deb K (2013)

Turning mechanisms for airborne LiDAR and photographic data acquisition. SPIE Journal of Applied Remote Sensing 7(1):1-19

[9]. Dashora A, Lohani B, \& Deb K (2013) Twostep procedure of optimization for solving the flight planning problem for airborne LiDAR data acquisition. International Journal of Mathematical Modelling and Numerical Optimization 4(4):323-350. 20.

[10]. Dashora A, Lohani B, \& Deb K (2012) Flight planning system for airborne data acquisition. 21. [11]. ASPRS (2011) ASPRS LAS format standard 1.4 .

[12]. Dashora A \& Lohani B (2013) LiDAR technology and a new method of flight planning for airborne LiDAR data acquisition. in ISRS - ISG Conference (Vishakhapattanam, India).
Harsh Jindal, Jagdeep kaur, "Airborne LiDAR", International Journal of Scientific Research in Science, Engineering and Technology (IJSRSET), Online ISSN : 2394-4099, Print ISSN : 2395-1990, Volume 8 Issue 3, pp. 495-498, May-June 2021. Available at doi : https://doi.org/10.32628/IJSRSET2183209 Journal URL : https://ijsrset.com/IJSRSET2183209 